\title{
Specimen temperature conditioning and drift before an S4 pipe fracture test
}

PS Leevers", S Hillmansen

(Department of Mechanical Engineering, Imperial College London, London SW7 2BX, UK)

and

\section{L de FF Moreno}

(University of Toronto, Department of Material Sciences and Engineering, Toronto, M5S 3E4, Canada)

\begin{abstract}
The resistance of plastic (primarily polyethlene) pipe to rapid axial crack propagation under pressure is specified using the ISO 13477 'S4' (small scale steady state) test method. Pipe performance in this test may be very sensitive to temperature under standard test conditions. There has recently been concern that the temperature conditioning of pipe specimens required by ISO 13477 may not be sufficiently precise. In order to compensate for an over-estimated increase in surface temperature between removal from the conditioning fluid and testing, some laboratories condition specimens well below test temperature. Here, infra-red imaging data are used to calibrate a simple and conservative model for the evolution of surface temperature during this interval. The results broadly support the present specification for testing at $0^{\circ} \mathrm{C}$, and provide a more secure basis for conditioning of specimens to be tested at lower temperatures.
\end{abstract}

*Communicating author (p.leevers@imperial.ac.uk, Fax 0207594 7017) 


\section{Introduction}

A large and increasing proportion of the pipe used for distributing fuel gas and potable water is extruded from polyethylene (PE). The PE grades used for this application must satisfy tight specifications on a number of criteria in a very competitive market, and they have evolved to a high level of performance by gaining in structural complexity. Because these materials are used in thick sections, plane strain fracture is a real threat. Full scale tests on air-pressurised pipe, using a method now standardised as ISO 13478, have shown that a crack running axially at sufficient speed $-100-300 \mathrm{~m} \mathrm{~s}^{-1}-$ can settle into a dynamic equilibrium with the decompression which it causes. Once initiated, this Rapid Crack Propagation (RCP) can therefore continue indefinitely if the initial pressure is high enough and the temperature is low enough.

Since correlation of RCP resistance to other material properties (e.g. impact strength) remains uncertain, specifying standards for pressure pipe require that it be measured 'as extruded', using the ISO 13477 S4 (small-scale steady state) test. Though certainly small compared to the full-scale reference method ISO 13478, the scale of S4 test specimens may be inconveniently large compared to that of human beings. The required 7-diameter length of 250 mm diameter polyethylene pipe of SDR 11 (i.e. whose nominal Standard Dimension Ratio of outside diameter to wall thickness is 11) weighs more than $25 \mathrm{~kg}$.

To add to the difficulties of handling such specimens before test, current specifications, which were originally designed to match the Northern European market for buried gas distribution systems, require them to be tested at $0^{\circ} \mathrm{C}$. Some $\mathrm{S} 4$ test installations have used in situ temperature conditioning. However, most laboratories condition specimens in a separate unit 
and then transfer them to a pressurising rig at ambient temperature. In order to avoid unacceptable temperature drift, ISO 13477 restricts the interval between removal from the conditioning fluid and testing to 3 minutes. The result can be a rushed step in the procedure, and there is increasing concern that $\mathrm{S} 4$ test results for temperature-sensitive grades of PE pipe could be significantly in error.

Some pipe grade PEs certainly show significant sensitivity to temperature. Most currently installed pipe is extruded from materials of strength class PE80. These show a (pipe size dependent) brittle-tough transition at $10-20^{\circ} \mathrm{C}$; thus PE80 pipes are tested for use on the brittle side of the transition, where they still have adequate toughness. However, much of the polyethylene pipe currently being installed is extruded from polyethylene of strength class PE100. The greater structural complexity of PE100 grades gives them exceptional resistance to RCP but renders them more sensitive to variations both in synthesis parameters and in processing history. PE100 resins show - and must continue to show - a ductile-brittle transition at temperatures typically $30 \mathrm{~K}$ lower than that of PE80, i.e. at -20 to $-10^{\circ} \mathrm{C}$. Because the transition must remain below the conventional test temperature, the test temperature must be accurate.

Because PE100 resins seldom show RCP in the $\mathrm{S} 4$ test at $0^{\circ} \mathrm{C}$, they can only be assessed quantitatively using the alternative critical temperature measurement procedure. This is written into ISO13477 as Annex B. A representative pressure is now chosen and tests are carried out at temperatures below $0^{\circ} \mathrm{C}$ until the arrest/propagation limit is found. As PE resins improve, test temperatures will inevitably decrease, critical temperature specifications will inevitably replace critical temperature specifications and the difficulties of handling the specimens before testing will multiply. 
Recently, concern has grown that the ISO 13477 procedures for temperature conditioning, and the 3-minute maximum limit on post-conditioning set-up time, are inadequate even for $0^{\circ} \mathrm{C}$ testing. PE100 resins gain much of their fracture resistance from the plane-stress surface layer which warms up most quickly; they therefore have most to gain from adventitious warming of the surface. This makes makes the conditioning issue commercially as well as technically contentious.

The work reported here was prompted by another (unpublished) study in another laboratory. Using readings from thermocouples mounted on S4 pipe specimen surfaces or buried within the wall, the investigators concluded that temperature drift during the 3 minute setup period for an $0^{\circ} \mathrm{C} \mathrm{S} 4$ test was far greater than had been supposed. They concluded that much colder initial conditioning - e.g. $-10^{\circ} \mathrm{C}$ for $250 \mathrm{~mm}$ SDR11 pipe - was needed in order to compensate for this drift. At Imperial College London, we maintained a consistent policy of cooling to $2^{\circ} \mathrm{C}$ below the nominal test temperature. The present study uses calculations from simple one-dimensional heat conduction analysis, calibrated using experimental measurements by infra-red imaging, to investigate the problem. With revision of ISO13477 imminent, our objectives have been to review the ' 3 minute rule' and the temperature tolerances on initial conditioning and to set up a rational basis for improving or replacing them if necessary.

\section{S4 test conditioning procedure under ISO13477}

Section 9 of ISO 13477 deals with procedures for bringing the test specimen pipe to a uniform temperature which is sufficiently close to the test temperature. Its requirements are in some respects a little vague. The section begins by stating that whatever test temperature required by the specification '... shall usually [our italics] be attained by immersion in a conditioning fluid 
maintained at the test temperature, to within ${ }_{-2}^{0}{ }^{\circ} \mathrm{C}$.' This fluid must not affect the properties of the pipe and clearly air, or water, are the conditioning fluids envisaged. A minimum immersion time is set by reference to another standard (ISO 1167), and it can safely be assumed to have brought the specimen to thermal equilibrium.

Unfortunately, the 'usually' in the first sentence corrupts its intended meaning, since there is no specific guidance as to what the final state of the pipe should be. ISO 13477 does goes on to specify that "All necessary precautions shall be taken to ensure that no significant [our italics] increase in the temperature of the test pipe occurs prior to testing. Crack initiation shall follow within 3 min of removal of the test pipe from the conditioning fluid." It is clearly intended that the pipe should be cooled to a homogeneous state near the test temperature, and that the temperature field should remain both reasonably homogeneous and reasonably well characterised by the nominal test temperature. However, the low thermal diffusivity of PE makes these conditions difficult to achieve simultaneously after 3 minutes at ambient temperature even for a $0^{\circ} \mathrm{C}$ test, and ISO 13477 is written to permit testing at much lower temperatures.

As a note within ISO13477 hints, the 3-minute maximum set-up time was arrived at empirically during development of the S4 test method [1]. Direct readings from thermocouples buried at mid-wall plane in $250 \mathrm{~mm}$ PE pipe showed only a very small temperature increase. It was reasoned that the mid-wall temperature characterises the overall temperature distribution, since the warmer surface layers remain thin. Perhaps because setting up an S4 test on a heavy, remotely conditioned $250 \mathrm{~mm}$ specimen in less than 3 minutes is not easy, laboratories have not rushed to investigate whether it should be set up even more quickly. 
Experience with the S4 test has shown that the ductile-brittle transition in PE100 materials is strongly influenced by the properties of the surface layers, particularly that at the bore [2]. The evidence is not clear-cut. Since it is difficult to grade through-thickness properties, we have tested specimens scored with internal and external axial surface notches [3] to remove the plane stress surface layer. Bore notches have a significant and deleterious effect, which is why ISO 13477 sanctions their use in the S4 specimen crack-initiation region. However, axial boresurface notches control the crack because the crack chooses to follow them, and this is generally not the case for external notches. The case for care in controlling bore-surface conditions is strong; the case for less care for the external surface is not.

It might seem obvious to check the surface temperature of the pipe immediately before testing and to specify an acceptable tolerance with respect to the nominal test temperature. In practice, this is very difficult to achieve accurately. Because the low thermal diffusivity of PE is so low - about $2 \times 10^{-7} \mathrm{~m}^{2} \mathrm{~s}^{-1}-$ thermal equilibrium is restored only very slowly after contact with a surface thermometer. The likely result is a suspect reading, a delayed test, or both.

Attaching a thermocouple on or just below the surface is equally dubious. In a warmer environment, thermocouple lead-out connections conduct heat inwards, giving an artifically high temperature reading. It was a thermocouple technique which had been used to obtain the alarming results referred to above. The results, and an over-simplified thermal analysis which ignored the influence of diffusivity on transient thermal response, had been used to determine a surface heat transfer coefficient of $h=20 \mathrm{~W} \mathrm{~m}^{-2} \mathrm{~K}^{-1}$. This $h$ value, in turn, was used to derive 
nomographs specifying the cooling conditions needed to achieve $0^{\circ} \mathrm{C}$ surface temperature at the moment of testing for various sizes and thicknesses of pipe.

The investigation reported here followed the same overall strategy, but used an experimental technique better suited to the surface properties of PE and a thermal analysis more representative of the bulk properties. A more thorough (but much more tedious) investigation would involve repeated evaluations of critical temperature with various set-up times. It is hoped that results reported here could provide a satisfactory basis for planning such a programme in the future.

\section{Experimental procedure and results}

Tests were carried out on black and yellow specimens of 180 and $250 \mathrm{~mm}$ diameter pipe. These were cooled to a nominal temperature of $-2 \pm 0.5^{\circ} \mathrm{C}$ and brought out into a laboratory atmosphere at $23^{\circ} \mathrm{C}$. Internal and external surface temperatures were then monitored using a FLIR Systems Agema Thermovision 510 infra-red camera.

Care was taken to monitor the bore surface of these large specimens conservatively while avoiding any unrepresentative forced convection. The specimen was cut to 3.5 diameters in length (i.e. to one-half of the standard S4 specimen length) and an axial convection symmetry condition was imposed by sealing one end of the bore with polystyrene foam (Fig. 1). A 50 $\mathrm{mm}$ diameter radial 'port-hole' was cut through the wall half-way along this half-length specimen, so that the opposite bore surface could be viewed. After temperature conditioning, the specimen was positioned with its axis horizontal, its ends resting on polystyrene foam blocks and its port-hole on top. The thermal imaging camera always pointed downwards. In 
this way the external surface was subject to convection in still-air conditions representative of the crack path in an S4 test - which is normally on top.

The intensity of an infra-red image depends on the emissivity of the viewed surface as well as on its temperature. The camera was therefore calibrated separately for each material tested. Low temperature calibrations were carried out by imaging a compression-moulded plaque inside a top-opening conditioning unit. The top was opened, the specimen was immediately imaged from above, and the temperature was recorded using a mercury-in-glass thermometer next to it. Higher temperature calibrations were made using a specially constructed rig in which two flat plates of polymer were heated between a pair of electrical heating pads. The plaque temperature was controlled by feedback from a thermocouple sandwiched between them. When the required temperature had stabilised, the plates were separated and were immediately imaged.

During the main S4 set-up imaging tests, thermal images were recorded every 30 seconds as 8bit deep monochromatic bit maps, each about $300 \mathrm{kB}$ in size. The longest test, reported here, involved recording over 900 images. Figure 3(a) shows the first of these. At this point the pipe is at uniform temperature. However the port-hole is just visible thanks to thin whiskers of PE left when machining it; these have quickly warmed up to appear as a 'corona'. After 1000 s (Fig 3(b)) - more than 5 times the 3 minute setup period - it is already evident that only the sharp edges of this artificial feature are showing significant increase in temperature. Only after $8000 \mathrm{~s}$ (Fig. 3(c)) has the outside of the pipe begun to approach room temperature, but even at this stage the bore surface remains much colder - in fact the temperature difference between bore and outside surfaces has reached its maximum. At the end of the test the image field has degenerated to a uniform grey and the pipe is barely distinguishable. Note that each 
thermal image is time-stamped; this experiment began at 17.32 and ran automatically overnight.

Images were then processed automatically, after appropriate windows on the image field for pipe bore, pipe exterior and control temperature surfaces had been identified. An average image intensity was recorded for each of the three windows on each image. Non-responsive pixels on the charge-coupled infra-red detector array, which are evident as white spots on Fig. 3 , were identified and excluded individually from the average. These average intensities were converted from arbitrary integer bitmap intensities to temperatures using the appropriate calibration. The images were also concatenated into a movie which was reviewed visually.

Figure 4 shows bore and outside surface temperatures as functions of time. The indicated bore surface temperature increases very slowly indeed: in fact for nearly 5000s, the image seen within the port-hole shows hardly any resolvable change in intensity. The outside surface warms up at a rate which, though much higher than the bore surface, is still very low on a 3 minute timescale.

\section{Analysis}

It quickly became clear that the surface temperature was increasing much more slowly than the earlier thermocouple experiments had led us to fear. However, although the thermal imaging system still had satisfactory linearity towards the lower end of its range (which was nominally -5 to $+100^{\circ} \mathrm{C}$ ), the images contained significant noise. It was difficult to resolve temperatures to better than $\pm 1^{\circ} \mathrm{C}$. Instead of relying directly on experimental data for a $180 \mathrm{~s}$ period, data from long-duration tests were therefore fitted globally to extract the surface heat transfer 
coefficient. The straightforward analysis outlined below uses this measured value to model the effect of set-up time on surface temperature for other pipe sizes and conditioning temperatures. For simplicity, a first-order, linear thermal conduction analysis is used, although in fact the thermal properties of $\mathrm{PE}$ vary considerably within the -30 to $+23^{\circ} \mathrm{C}$ range of interest for pipe testing. Property data used in the calculation were: mass density $\rho=940 \mathrm{~kg} \mathrm{~m}^{-3}$, specific heat $c_{\mathrm{p}}=1.9 \mathrm{~kJ} \mathrm{~kg}^{-1} \mathrm{~K}^{-1}$, thermal conductivity $k=0.35 \mathrm{~W} \mathrm{~m}^{-1} \mathrm{~K}^{-1}$. The high thermal conductivity of crystalline PE gives this polymer a thermal diffusivity nearly twice as high as that typical of other polymers. We have adopted a $0^{\circ} \mathrm{C}$ reference state both as a standard test condition and as an average state between a typical laboratory environment and a typical low-temperature 'critical temperature' test.

Some further approximations yield a tractable model. The pipe wall is treated as an infinite flat slab of constant thickness $2 L$. The two slab surfaces are assumed to have the same thermal conductivity $k$, but the surface heat transfer coefficient has a constant value of $h$ on one surface and of $r h$ on the other where $0<r<1$ is a parameter. End effects are neglected and the difference in area between the bore and outside diameter surfaces is lumped into the surface heat transfer coefficient. The specimen, after having reached thermal equilibrium at a conditioning temperature $T_{0}$, is assumed to be immersed at time zero in an environment at constant temperature $T_{\mathrm{amb}}$, to which it loses heat from both surfaces.

Carslaw and Jaeger [4] present the solution to this problem in the form

$$
T_{\text {amb }}-T=\sum_{n=1}^{\infty} Z_{n}(x) \exp \left(-\kappa \beta_{n}^{2} t\right) \int_{0}^{2 L} Z_{n}\left(x^{\prime}\right) \mathrm{f}\left(x^{\prime}\right) \mathrm{d} x^{\prime}
$$

where the coordinate $x$ varies from zero on the inner surface to $2 L$ on the outer surface. In this case the initial temperature distribution is 


$$
T_{\text {amb }}-T=\mathrm{f}\left(x^{\prime}\right)=\left(T_{\mathrm{amb}}-T_{0}\right)=\text { const. }
$$

This solution can only be evaluated numerically. $Z_{n}$ is found by solving the eigenvalue equation

$$
Z_{n}(x)=\frac{\left[2\left(k^{2} \beta_{n}^{2}+r^{2} h^{2}\right)\right]^{1 / 2}\left(k \beta_{n} \cos \beta_{n} x+h \sin \beta_{n} x\right)}{\left\{\left(k^{2} \beta_{n}^{2}+h^{2}\right)\left[2 L\left(k^{2} \beta_{n}^{2}+r^{2} h^{2}\right)+k r h\right]+k h\left(k^{2} \beta_{n}^{2}+r^{2} h^{2}\right)\right\}^{1 / 2}}
$$

where $\beta_{n}$ is the $n$th positive root of

$$
\tan 2 \beta L=\frac{k h(1+r)}{\left(k^{2} \beta^{2}-r h^{2}\right)} \beta .
$$

On defining a Biot number $\mathrm{Bi}=\frac{2 L h}{k}$ and a dimensionless thickness $\delta=2 \beta L$ the eigenvalue equation reduces to

$$
\tan \delta=\frac{(1+r) \mathrm{Bi}}{\left(\delta^{2}-r \mathrm{Bi}^{2}\right)} \delta
$$

and on defining $\xi=\frac{x}{2 L}$ we find that Eqn. (3) reduces to the more convenient form

$$
Z_{n}(x)=\frac{\delta_{n} \cos \delta_{n} \xi+\mathrm{Bi} \sin \delta_{n} \xi}{\left(C_{n} L\right)^{1 / 2}}
$$

where

$$
C_{n}=\mathrm{Bi}+\left(\delta_{n}^{2}+\mathrm{Bi}^{2}\right)\left(1+\frac{r \mathrm{Bi}}{\left(\delta_{n}^{2}+r^{2} \mathrm{Bi}^{2}\right)}\right) .
$$

Hence, finally,

$$
\begin{aligned}
T^{*} & =2 \sum_{n=1}^{\infty} \frac{\delta_{n} \cos \delta_{n} \xi+\mathrm{Bi} \sin \delta_{n} \xi}{C_{n}} \exp \left(-\frac{1}{4} \mathrm{Fo}_{n}^{2}\right) \int_{0}^{1}\left(\delta_{n} \cos \delta_{n} \xi^{\prime}+\mathrm{Bi} \sin \delta_{n} \xi^{\prime}\right) \mathrm{d} \xi^{\prime} \\
& =2 \sum_{n=1}^{\infty} \frac{\delta_{n} \cos \delta_{n} \xi+\mathrm{Bi} \sin \delta_{n} \xi}{C_{n}}\left[\sin \delta_{n}+\frac{\mathrm{Bi}}{\delta_{n}}\left(1-\cos \delta_{n}\right)\right] \exp \left(-\frac{1}{4} \mathrm{Fo} \delta_{n}^{2}\right)
\end{aligned}
$$


Equation (8) was evaluated numerically. The computed results were verified using one of the many published standard tables, and an independent though similar program was used to solve the special case of symmetrical cooling. This second code provided two solutions for crosschecking against those from the more complicated asymmetrical case detailed above: symmetrical cooling $(r=1)$, and cooling from one surface with perfect insulation on the other $(r=0)$. The latter emerges from the symmetrical analysis as the mid-plane temperature within a slab $4 L$ thick.

By trial and error, the best fit for cooling conditions according to the model above was obtained by prescribing an external heat transfer coefficient $h=6.5 \mathrm{~W} \mathrm{~m}^{-2} \mathrm{~K}^{-1}$ and an $r$ value (i.e. an internal $h$ ) of zero. However, Fig. 4 emphasises that even these very low values do not do justice to the almost negligible cooling rate during the first few minutes of postconditioning set-up.

\section{Discussion}

Those whose business it is to extrude plastic pipe of these dimensions will not be surprised by our results. Post-extrusion cooling of thick pipe from about $200^{\circ} \mathrm{C}$ to a temperature low enough to avoid slump - typically $80^{\circ} \mathrm{C}-$ is a slow process even under forced convection. Within 'annealing zones' between water cooling tanks, the pipe cools by convection into still air under conditions similar to those studied here. Several attempts have been made to optimise the cooling process via computer simulation, and some have involved evaluation of surface heat transfer cofficients $h$.

Barnes [5] used infra-red thermal imaging to measure pipe surface temperatures on a $400 \mathrm{~mm}$ extrusion line, and match them with those output from a one-dimensional finite difference 
model. His value of $h=5 \mathrm{~W} \mathrm{~m}^{-2} \mathrm{~K}^{-1}$ was even lower than that measured here. BP Chemicals plc, developing a similar computational extrusion line model in the mid-1990s [6], arrived at slightly higher values of $h=8-15 \mathrm{~W} \mathrm{~m}^{-2} \mathrm{~K}^{-1}$ for the annealing zones, again using thermal imaging. Pittman et al. [7] determined $h$ values of $15-20 \mathrm{~W} \mathrm{~m}^{-2} \mathrm{~K}^{-1}$, for pipes of similar size to those investigated here, by attaching thermocouples to the surface of pipe passing along the line. The use of thermocouples to instrument materials of such low thermal diffusivity so far from thermal equilibrium systems is fraught with difficulties. A recently initiated study of thermal measurement technology for plastics processing, at the UK National Physical Laboratory [8], has shown the importance of siting thermocouple leads along isotherms to minimise thermal conduction along them.

The natural convection component of surface heat transfer from a long horizontal cylindrical surface at temperature $T$ can be estimated using textbook analysis (e.g. [9]). The Prandtl number for this process is low (0.76) and the Nusselt number $N u=h D / k$ can be estimated from the correlation

$$
N u=0.52 R a^{1 / 4}
$$

where the Rayleigh number $R a$ is given in terms of air density $\rho$, specific heat $c_{\mathrm{p}}$, viscosity $\mu$ and thermal conductivity $k$ by

$$
R a=\frac{\rho^{2} g c_{\mathrm{p}}}{\mu k} \frac{\left(T-T_{\mathrm{a}}\right)}{\bar{T}} D^{3}
$$

$g$ being gravitational acceleration and $\bar{T}$ the mean absolute temperature of the boundary layer. Re-arrangement yields

$$
h=0.52\left[\frac{\rho^{2} g c_{\mathrm{p}} k^{3}}{\mu D} \frac{\left(T-T_{\mathrm{a}}\right)}{\bar{T}}\right]^{1 / 4}
$$

and substituting property values for air we arrive at the estimate 


$$
\tilde{h}=4.2\left(\frac{D_{\text {ref }}}{D}\right)^{1 / 4} \mathrm{~W} \mathrm{~m}^{-2} \mathrm{~K}^{-1}
$$

for a reference diameter $D_{\text {ref }}=250 \mathrm{~mm}$ and a $0^{\circ} \mathrm{C}$ test temperature. For $D=D_{\text {ref }}$ this estimate is in satisfactory agreement with the $6.5 \mathrm{~W} \mathrm{~m}^{-2} \mathrm{~K}^{-1}$ measured, but it is lower. Pittman et al. [7] recognised that their extrusion line $h$ values, which were derived from thermocouple data and were even higher than those reported here, exceeded those expected from free convection. The excess may partly be attributable to evaporative cooling [10], since the pipe emerges hot and wet from the preceding spray tank. A higher measured value would be expected anyway, since radiant heating from the environment has not been accounted for. It is very difficult to hazard an estimate for this contribution, but if it were of major significance then it would show up as a dependence of cooling rate on pipe colour - which neither we nor Pittman [7] were able to distinguish. On this basis Eqn. (11) is corrected to account for the diameter dependence of $h$ as

$$
h=2.3+4.2\left(\frac{D_{\text {ref }}}{D}\right)^{1 / 4} .
$$

Turning now to results for the bore surface, it is seen that even setting $h=0-$ which Beech [4], too, found necessary in order obtain agreement between observation and calculation - our measurements do not correspond to the free convection model. This is because the model assumes the surface to be in contact with the ambient environment, which is clearly unrealistic for a pipe specimen of this length. Even if the original charge of conditioned air spills out of the pipe, the bore surface refrigerates a boundary layer of the laboratory air which replaces it. The contained air volume as a whole will certainly be colder than ambient air, and the bore surface cannot 'see' sources radiating to it from the environment. Internal free convection can do no more than ensure that the bore surface remains coldest where it is exposed to view by the port-hole. In summary, an $h=0$ boundary condition is physically reasonable and should even be conservative. 
Given our objectives, we preferred to stay with a very conservative model than to search for a more accurate one. Using the heat transfer parameters derived above, Fig. 5 plots temperature as a function of time for the first five minutes in laboratory air after conditioning for 110,180 and 250 SDR 11 PE pipe. Using Eqn. (12) the surface heat transfer characteristic $h$ has been increased as a function of diameter $D$ from $6.5 \mathrm{~W} \mathrm{~m}^{-2} \mathrm{~K}^{-1}$ for $250 \mathrm{~mm}$ pipe to 6.9 and $7.5 \mathrm{~W} \mathrm{~m}$ ${ }^{2} \mathrm{~K}^{-1}$ for 180 and $110 \mathrm{~mm}$ pipe respectively.

Figures 5(a) to (c) clearly reveal that the pipe wall thickness has only a very secondary influence on the outside surface temperature. The evolution of temperature is determined by the rate at which the surface can lose heat to the environment, which it does at a rate which depends little on diameter. The internal surface is more affected since the model allows it to lose heat only by conduction; the rate of temperature change is determined by the boundary (outside surface) temperature and the conduction distance, i.e. the thickness. Only for the thinnest pipe does the surface temperature just drift out of the required $\pm 1^{\circ} \mathrm{C}$ tolerance within the 3 minute setup time limit already specified by ISO 13477. It should again be recalled that the simulation relates very conservatively to the measured bore surface temperature.

The pipe bore surface may well warm up more by forced convection during air pressurisation immediately prior to an S4 test than it could do during our experiments. However, there is a wide safety margin to erode before the results of the model become pessimistic. Pressurisation usually occupies only a small fraction of the total set-up time (and could therefore only influence a very thin surface layer). Imperial College London have adopted the practice of pressurising S4 specimens from high-pressure bottled air which has inevitably cooled to a subambient temperature during depressurisation. 
On the other hand, no attempt has been made here to reduce the heat transfer artificially. While it is best not to make standard procedures any more prescriptive than necessary, various methods could be used in practice to preserve the conditioning temperature more accurately. Depending on the details of the test apparatus, it may be possible to skin specimens with polystyrene foam on external and even internal surfaces. It is certainly desirable to pressurise the pipe specimen in such a way that axial air currents at the bore surface are avoided.

Finally, Fig. 6 shows the conditioning temperature $T_{\text {cond }}$ needed to achieve a specified outside surface temperature (i.e. a nominal test temperature $T$ at an ambient temperature of $23^{\circ} \mathrm{C}$ ) after a 3-minute set-up time. This curve is essentially independent of pipe thickness or diameter, and it can be summarised using the simple equation

$$
T_{\text {cond }}=T+C\left(T-T_{\text {amb }}\right)
$$

where for $T_{\mathrm{amb}}=23^{\circ} \mathrm{C}, C=0.12 \mathrm{~K}^{-1}$ so that

$$
T_{\text {cond }}=1.12 T-2.8^{\circ} \mathrm{C} \text {. }
$$

\section{Conclusions}

The surface temperatures of pipe specimens simulating those used for the S4 rapid crack propagation test have been investigated using remote thermal imaging. The results provide reassurance that previous measurements of temperature drift during the prescribed 3-minute setup period were too high. A conservative estimate of the temperature increase for $250 \mathrm{~mm}$ pipe can be arrived at by assuming the exterior surface to radiate at $6.5 \mathrm{~W} \mathrm{~m}^{-2} \mathrm{~K}^{-1}$ into fluid at the ambient temperature, and the bore surface to be perfectly insulated.

\section{Acknowledgements}


The authors wish to thank Azmi Ashari, an undergraduate student of Electrical Engineering at Imperial College London, who obtained the experimental results while on placement with us under the Undergraduate Research Opportunities Programme (UROP). 


\section{References}

1 Yayla P, Leevers PS (1992). Rapid crack propagation in pressurised plastic pipe. II: Critical pressures for polyethylene pipe. Engng Fracture Mech 1992;42(4):675-682.

2 Leevers PS, Yayla P. In: Proceedings of the 12th Plastic Fuel Gas Pipe Symposium, Boston, 1991.p.58-69

3 Moreno LdFF. PhD thesis. U London. 2002

4 Carslaw HS, Jaeger JC. Conduction of Heat in Solids. 2nd ed. Oxford University Press. 1959.

5 Barnes, CM. The Cooling and Solidification of Thermoplastic Polymers. PhD Thesis, University of Bradford, Bradford, UK. 1989.

6 Beech SH, Personal Communication 2003.

7 Pittman JFT, Farah, IA, Isaac, DH, Eccott, A. Spray cooling heat transfer coefficients in plastic pipe manfacture. Plastics Rubber Comps. Proc. Applications 1995;24:123-129.

8 Brown C., Personal Communication, 2003.

9 Rohsenow WM, Hartnett, JP, Cho, YI. Handbook of Heat Transfer. 3rd ed. McGraw Hill. 1998.

10 Pittman JFT, Personal Communication, 2003. 


\section{List of figure captions}

1 Experimental arrangement for monitoring surface temperature of a pipe specimen

2 Thermal image temperature calibration for a yellow PE pipe material

3 Thermal images of a yellow $250 \mathrm{~mm}$ SDR 11 PE pipe (a) immediately, (b) $1000 \mathrm{~s}$ and (c) $8000 \mathrm{~s}$ after removal from the conditioning environment

4 Temperature as a function of time for a $250 \mathrm{~mm}$ SDR 11 pipe specimen conditioned at $2{ }^{\circ} \mathrm{C}$

5 Predicted surface temperature change during the set-up period for (a) $250 \mathrm{~mm}$, (b) 180 $\mathrm{mm}$ and (c) $110 \mathrm{~mm}$ SDR 11 pipe specimens

6 Conditioning temperature required to meet a specified test temperature after a 3 minute set-up time 


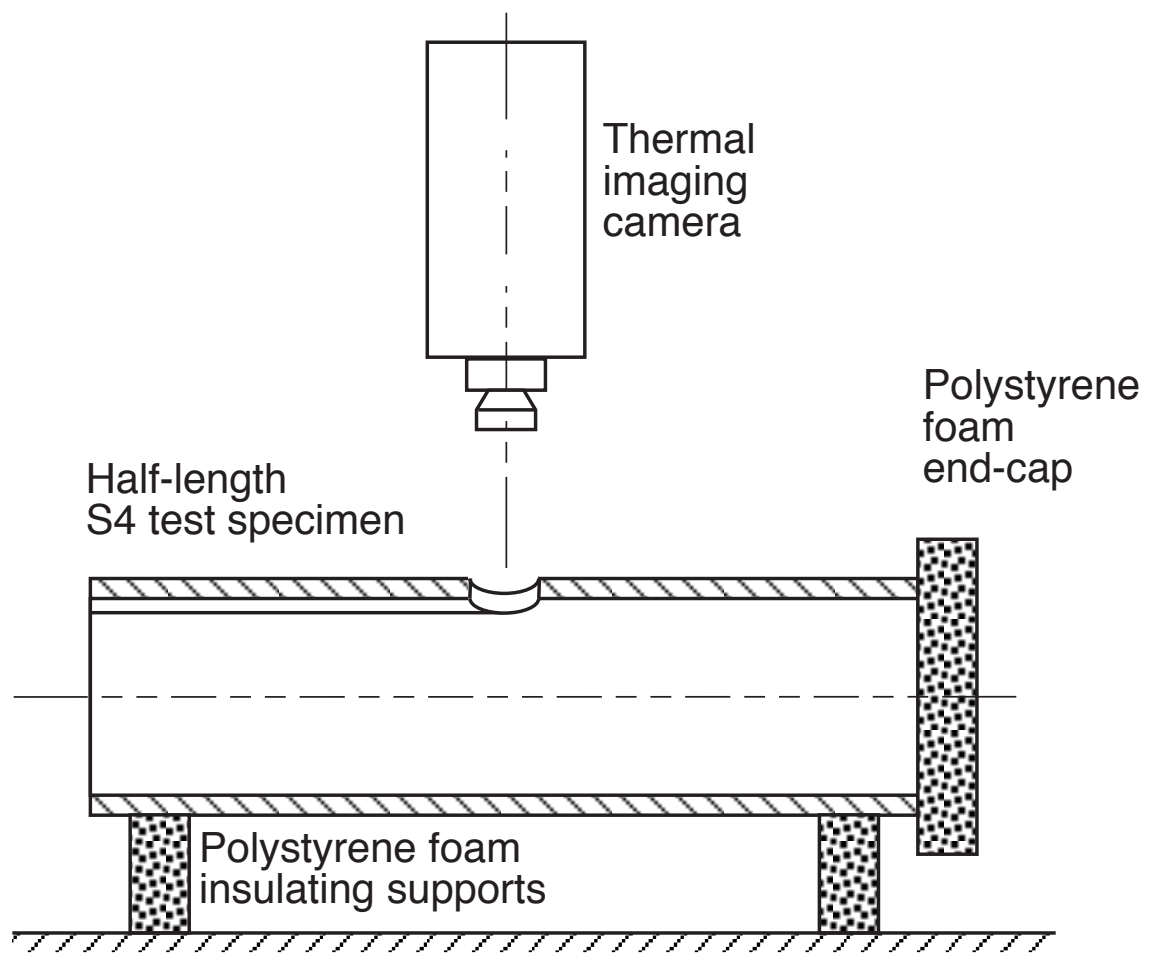




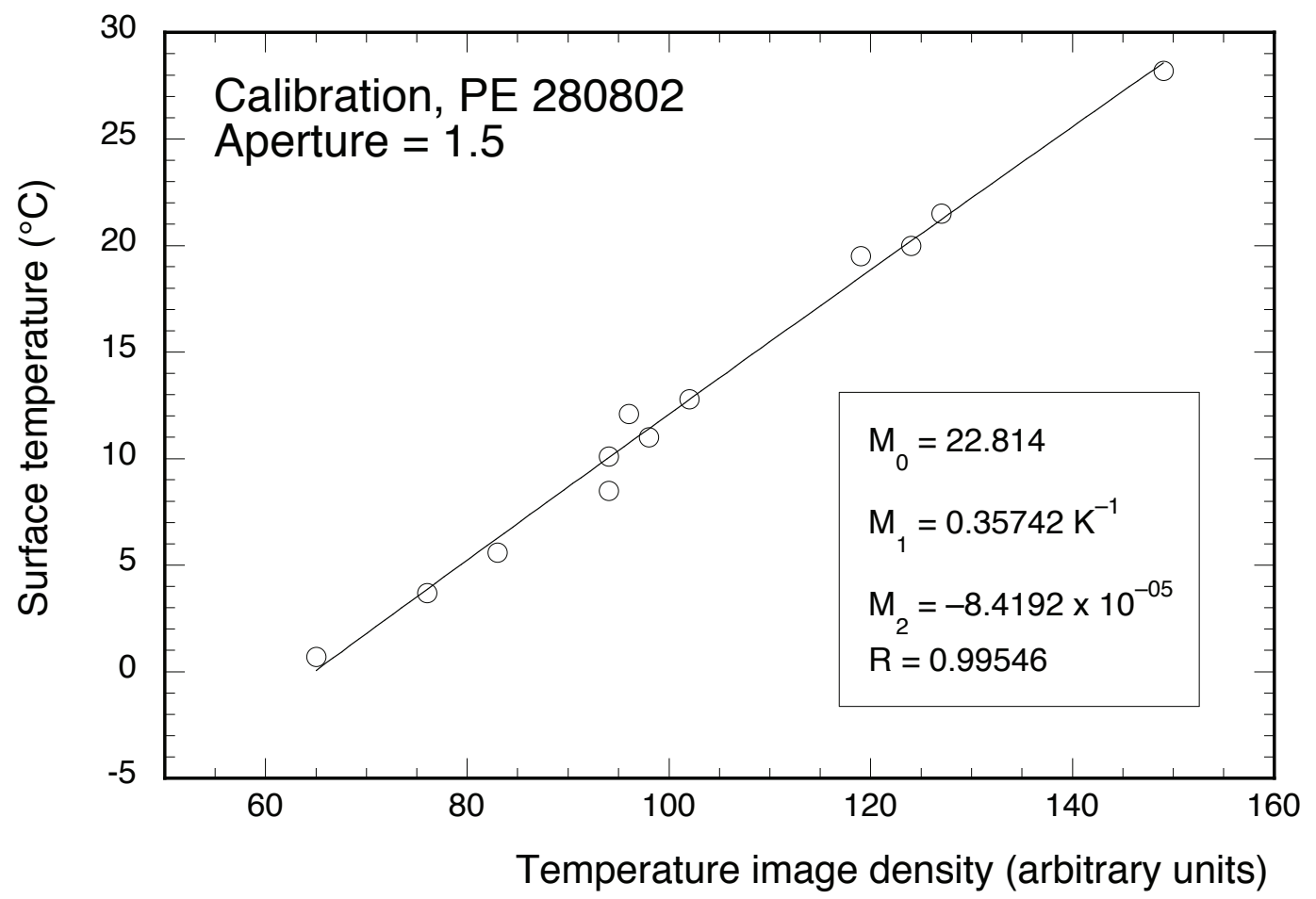



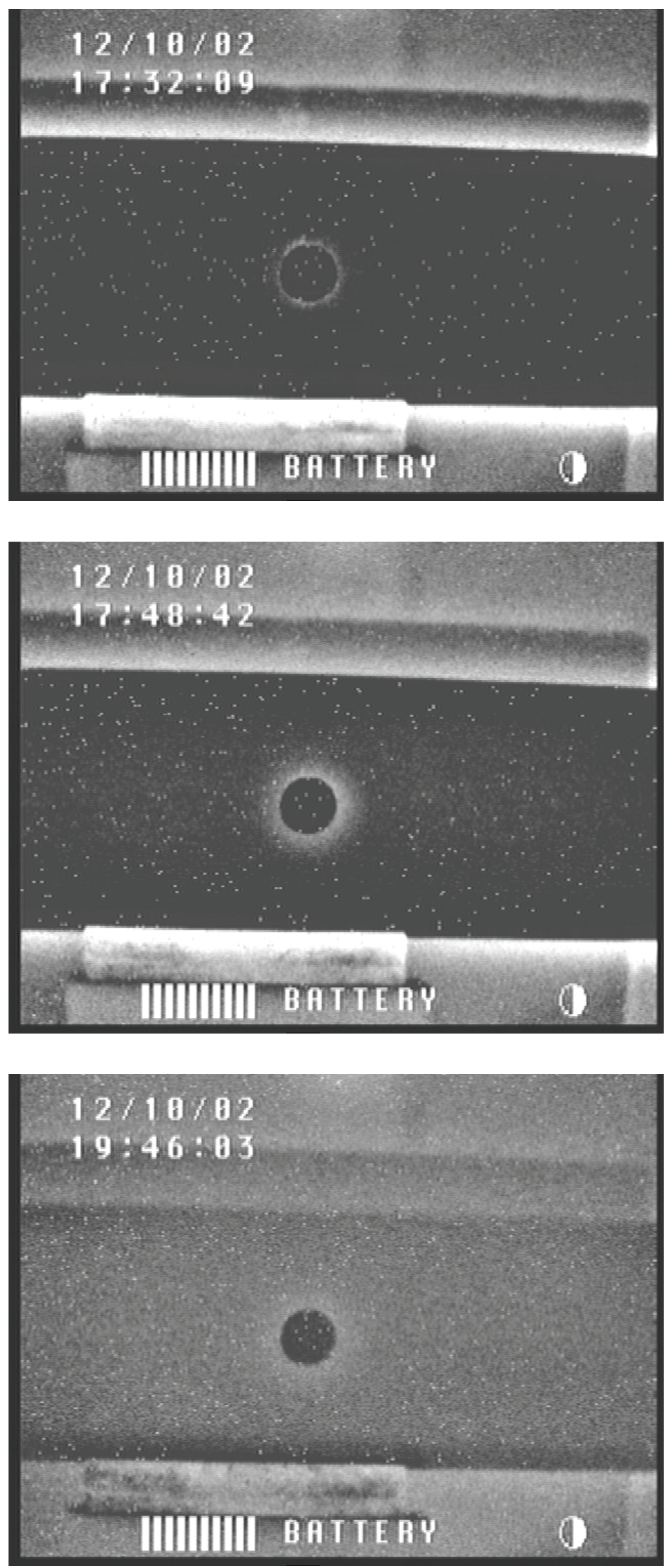


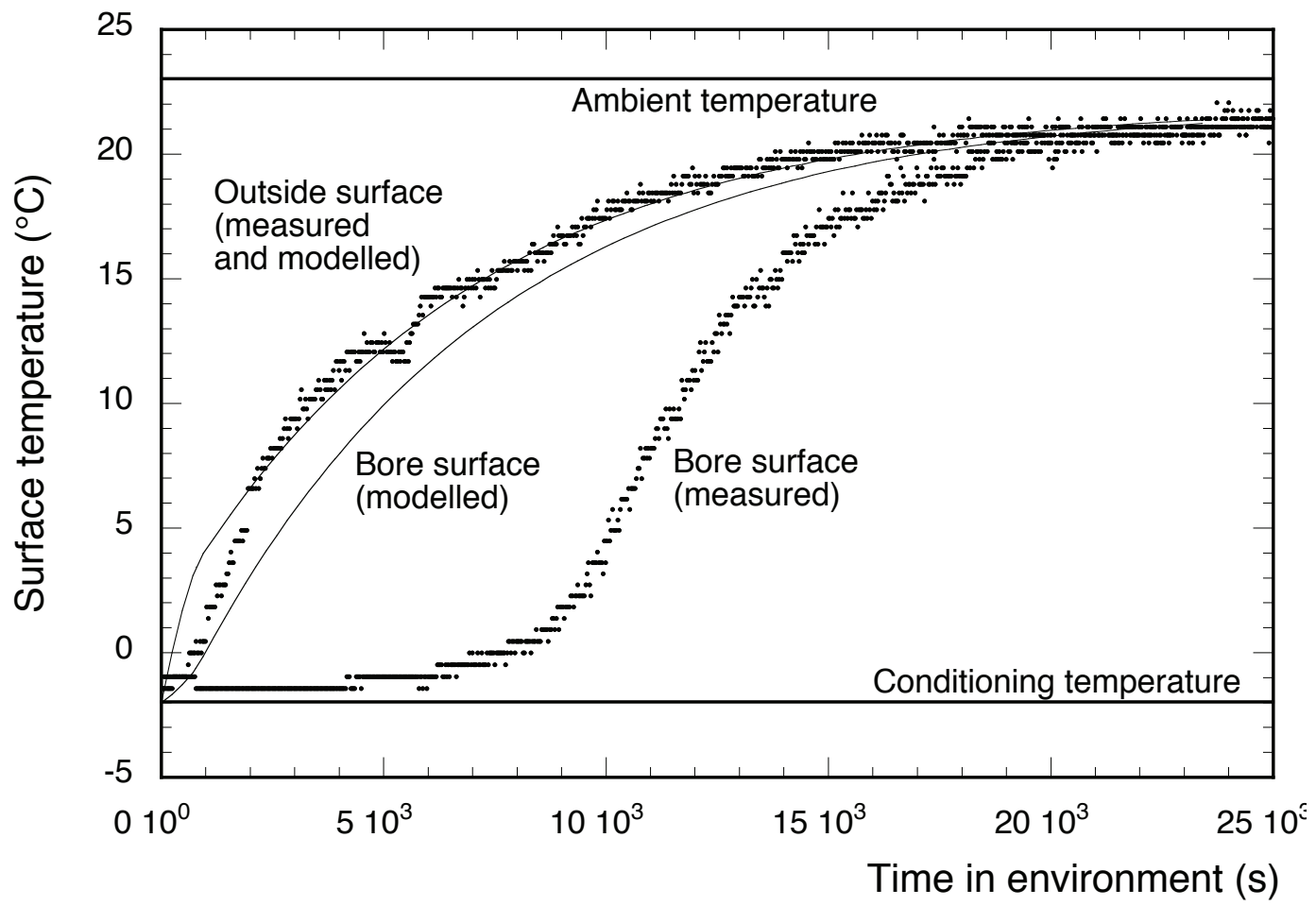



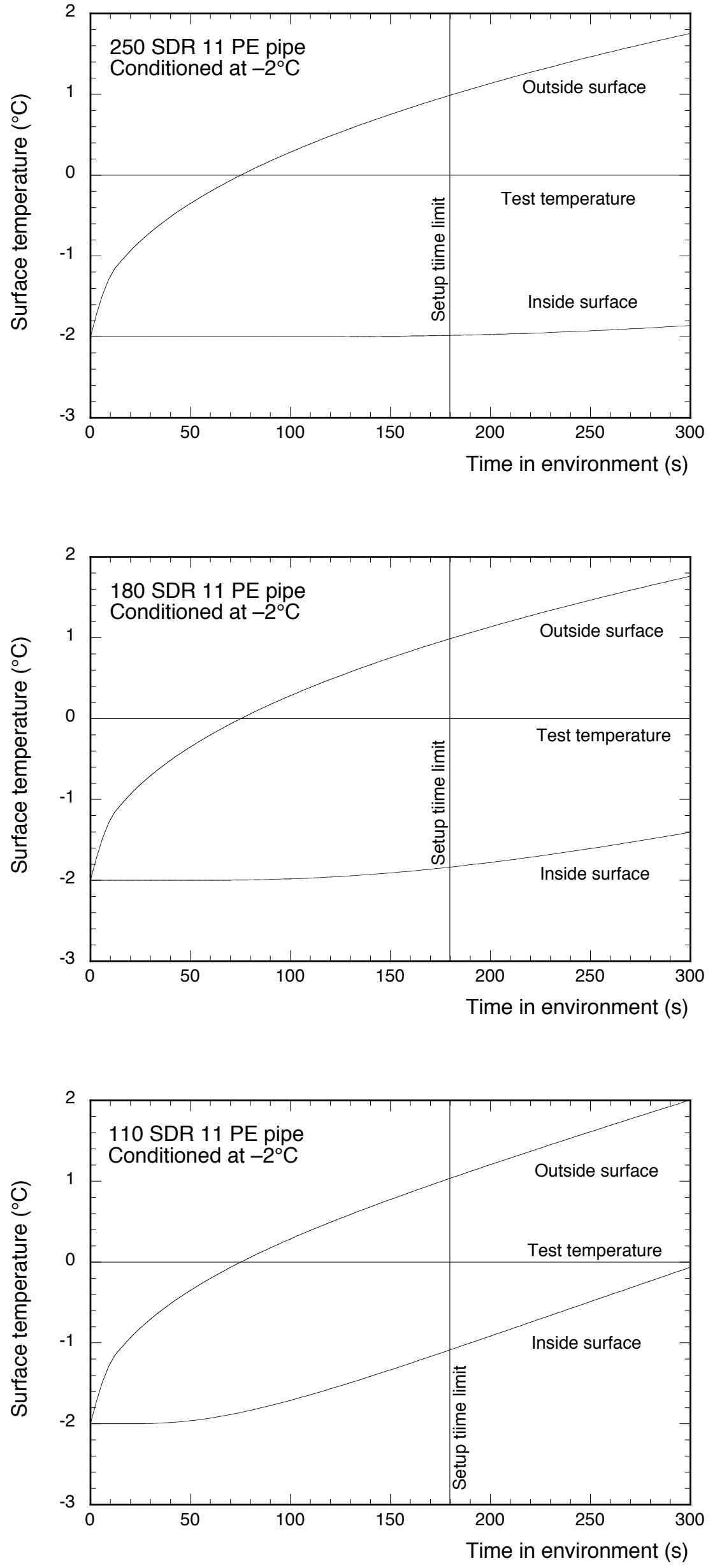


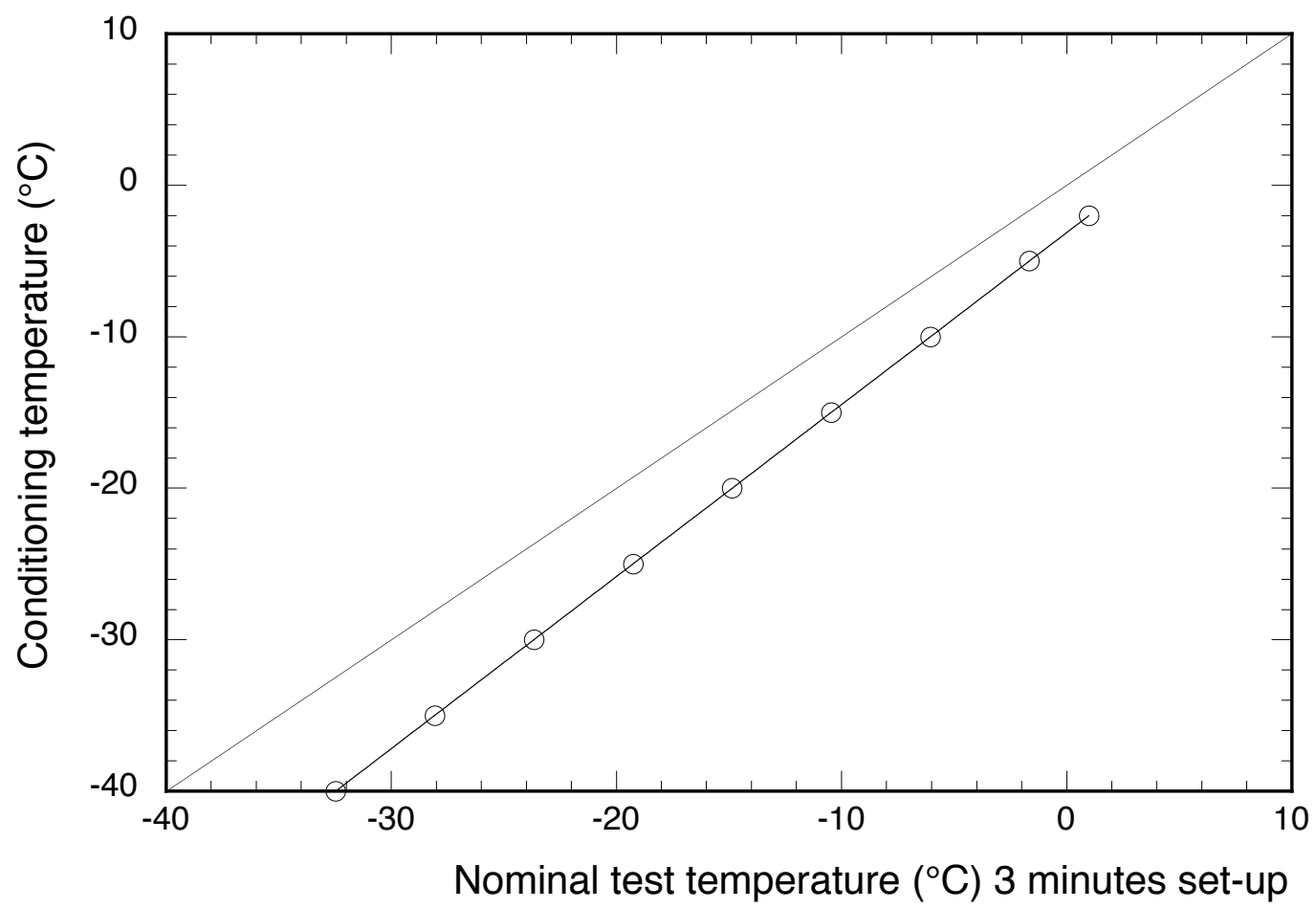

